\title{
Editorial
}

\section{Smart Manufacturing Technology}

\author{
Michele Calì (D)
}

Citation: Calì, M. Smart

Manufacturing Technology. Appl. Sci. 2021, 11, 8202. https://doi.org/ 10.3390/app11178202

Received: 26 July 2021

Accepted: 18 August 2021

Published: 3 September 2021

Publisher's Note: MDPI stays neutral with regard to jurisdictional claims in published maps and institutional affiliations.

Copyright: (C) 2021 by the author. Licensee MDPI, Basel, Switzerland. This article is an open access article distributed under the terms and conditions of the Creative Commons Attribution (CC BY) license (https:/ / creativecommons.org/licenses/by/ $4.0 /)$.
Department of Electrical, Electronics and Computer Engineering (DIEEI), University of Catania, 95124 Catania, Italy; michele.cali@unict.it

\begin{abstract}
This Special Issue of Applied Sciences provides a collection of original papers on smart manufacturing technology with the aim of: examining emerging aspects of digitalization in the industrial and biomedical fields, as well as in business management and sustainability; proposing and developing a new approach useful for companies, factories, and organizations to achieve greater innovation and productivity — as well as sustainability-by applying smart manufacturing technologies; and exploring new ideas and encouraging research directions so as to obtain autonomous and semiautonomous processes, high-quality products, and services with a greater integration and interconnection of resources while reducing costs. The advantages of new methods and experimental results obtained in the collected contributions are discussed promoting further design, implementation, and application in the various fields.
\end{abstract}

Keywords: sustainability; machine tools and manufacturing equipment; machine learning; computational geometry and CAD/CAM; assessment of digitalization; virtual/augmented reality; enabling technologies; manufacturing networks and security

\section{Introduction}

Smart manufacturing is a real industrial revolution-also called the Fourth Industrial Revolution or Industry 4.0, depending on the environment and context-that has transformed the manufacturing sector in recent years, mainly thanks to the development of digitalization.

The concept of smart manufacturing indicates a vision according to which, thanks to digital technologies, manufacturing companies will be able to increase the interconnection and cooperation of resources, both within plants and along the supply chain, with beneficial results for competitiveness and efficiency. Therefore, it can be stated that smart manufacturing constitutes the set of technologies and processes which allows resources to be utilized in a smarter and more "connected" way, thus aiming at obtaining increased speed and flexibility in manufacturing [1-4].

For some years now, companies have invested in many smart manufacturing technologies such as the Internet of things, Big Data, and cloud computing; advanced automation; wearable devices; and addictive manufacturing (3D printing) [5-7].

The main focus of this Special Issue is the latest advances made in interconnection and cooperation between the people, machinery, and information used during production processes, with the purpose of creating greater knowledge, facilitating better decision making, and reducing inefficiencies.

These advances cover novel manufacturing processes, manufacturing equipment, manufacturing systems and techniques, machine tools, and enabling technologies.

\section{The Special Issue Structure}

This Special Issue (SI) presents ten studies on new smart manufacturing technology and applications aimed at improving its actual knowledge and potentialities. More specifically, the following topics, listed in order of publication, are discussed: 
(1) A New Generation of Bio-Composite Thermoplastic Filaments for a More Sustainable Design of Parts Manufactured by FDM;

(2) Engineering and Manufacturing of a Dynamizable Fracture Fixation Device System;

(3) Utilization of Optimization of Internal Topology in Manufacturing of Injection Moulds by the DMLS Technology;

(4) Prediction of Model Distortion by FEM in 3D Printing via the Selective Laser Melting of Stainless Steel AISI 316L;

(5) Improvement of Delivery Reliability by an Intelligent Control Loop between Supply Network and Manufacturing;

(6) Solid-State Foaming Process Optimization for the Production of Shape Memory Polymer Composite Foam;

(7) A Five-Step Approach to Planning Data-Driven Digital Twins for Discrete Manufacturing Systems;

(8) A Study on Distance Measurement Module for Driving Vehicle Velocity Estimation in Multi-Lanes Using Drones;

(9) Product Quality Prediction for Wire Electrical Discharge Machining with Markov Transition Fields and Convolutional Long Short-Term Memory Neural Networks;

(10) Research on Fish Slicing Method Based on Simulated Annealing Algorithm;

(11) Secure and Privacy-Respecting Documentation for Interactive Manufacturing and Quality Assurance;

(12) Visioning the Future of Smart Fashion Factories Based on Media Big Data Analysis;

(13) Research on Temperature Compensation Method in Crankshaft Online Measurement System;

(14) HSV Color-Space-Based Automated Object Localization for Robot Grasping without Prior Knowledge.

Finally, the aim of this SI was to unite the researchers working hard in this field to produce the relevant theoretical, ideological, and empirical results using quantitative or qualitative approaches to technology.

\section{The Proposed Articles}

A brief description of the proposed manuscripts published is reported below:

The manuscript "A New Generation of Bio-Composite Thermoplastic Filaments for a More Sustainable Design of Parts Manufactured by FDM" by Michele Calì, Giulia Pascoletti, Massimiliano Gaeta, Giovanni Milazzo, and Rita Ambu focuses on the development of new composite materials through the fabrication of purpose-dedicated filaments. The study is an analysis of organic bio-plastic compounds made of polylactic acid (PLA) and organic by-products, representing a unique opportunity of symbiosis between the primary and secondary industries. Five new thermoplastic materials with fillers (HEMP, WEED, TOMATO, CAROB, and PRUNED) were considered suitable for biomedical applications thanks to their great versatility in design and efficiency. Their main physical, mechanical, and visual properties are described, along with two examples of their application in the biomedical field using additive manufacturing. Some main advantages of their use over classical competitors are discussed. The experiments concern the selection of two different biomedical devices - two prototypes of a neck orthosis and a laryngoscope designed and printed with the HEMP material - and the improvement in performance of these innovative thermoplastic materials with respect to standard ones thanks to their improved mechanical properties and weight reduction due to the specific filler used.

The study presented in the manuscript "Engineering and Manufacturing of a Dynamizable Fracture Fixation Device System" by Giancarlo Dichio, Michele Calì, Mara Terzini, Giovanni Putame, Elisabetta Maria Zanetti, Piero Costa, and Alberto Luigi Audenino is an investigation of the dynamization of an orthopedic plate for internal fracture fixation performed wirelessly and non-invasively in the post-operative period. The authors illustrate the peculiarities of the system design and manufacturing process, performed according to the respective Medical Device Regulation (EU) 2017/745, aimed at shortening 
healing times and enhancing the quality of the newly formed bone. The study discusses the development of a parametric multibody model, the evaluation of the fatigue strength of the device, and the static strength of the whole bone-plate system by finite element analysis and experimental testing of the dynamic plate performance on a reference fractured femur with excellent clinical results, as compared with the static fracture synthesis plates already available on the market.

In the manuscript "Utilization of Optimization of Internal Topology in Manufacturing of Injection Moulds by the DMLS Technology" by Tomas Coranic, Stefan Gaspar, and Jan Pasko, results regarding the creation of residual stresses in the manufacturing of molded parts for injection molding machines-using DMLS technology as an alternative solution to the monolithic model - are presented. The main interest of the DMLS process analysis is the reduction in residual stresses in the manufactured parts in order to ensure their proper function and durability. The authors propose to use the internal topology optimization of the part in combination with the appropriate orientation of the model in the workspace, unlike other research in the given field. The simulation results obtained indicated a reduction in residual stresses, a positive effect on mechanical properties, limitation of material consumption, and efficiency of time in the design of parts manufactured via DMLS technology, demonstrating that structural topology optimization can be applied to other methods of additive manufacturing.

The manuscript "Prediction of Model Distortion by FEM in 3D Printing via the Selective Laser Melting of Stainless Steel AISI 316L" by Marek Pagac, Jiri Hajnys, Radim Halama, Tariq Aldabash, Jakub Mesicek, Lukas Jancar, and Jan Jansa deals with metallic 3D printing technology in the field of stress prediction and simulation of printed components. The proposed method is the selective laser melting (SLM) method, used to verify the development of deformations during the removal of the part from the base plate using two software programs: ANSYS Additive Suite and MSC Simufact Additive. The study illustrates the performance of practical verification of the simulation of a 3D-printed topologically optimized part made of AISI 316L stainless steel and outlines the pros and cons of metallic 3D printing technology.

In the manuscript "Improvement of Delivery Reliability by an Intelligent Control Loop between Supply Network and Manufacturing" by Dennis Bauer, Thomas Bauernhansl, and Alexander Sauer, the development of a concept for closer connection between supply network and manufacturing through the integration of events from the supply network into manufacturing control's decisions on adapted schedules is proposed. The study deals with the discussion of a possible application of the concept, outlining its resulting obstacles and benefits with the aim of improving delivery reliability and resilience. The work is thought to be a contribution to the knowledge base in supply chain management, production planning and control (PPC), and organizational theory. As a result, the proposed approach leads to an effective and efficient response to events in the supply network through smarter manufacturing control, and thus more resilient manufacturing.

In the manuscript "Solid-State Foaming Process Optimization for the Production of Shape Memory Polymer Composite Foam" by Tamem Salah and Aiman Ziout, the focus is on the optimization of the solid-state foaming process of polyester-based thermoset polymers (polyester-based polymers $/ \mathrm{Fe}_{3} \mathrm{O}_{4}$ nanocomposite foaming). In the analyzed process, the foam ratio and density are identified as process performance indicators with the aim of obtaining the highest foaming ratio and the lowest foaming density for the prepared samples. The tests performed on two different polymers, namely CC and JSD, indicated the optimum parameters to prepare the highest foaming ratios. The results of the study demonstrate a greater tendency to reach higher foaming ratios and lower densities in the CC polymer powder as compared with JSD because of TGIC crosslinks within the matrix, which allow for better heat conductivity and easier motion.

The manuscript "A Five-Step Approach to Planning Data-Driven Digital Twins for Discrete Manufacturing Systems" by Matevz Resman, Jernej Protner, Marko Simic, and Niko Herakovic focuses on the problem of developing digital models that form the digital 
twin connected with a real manufacturing system in order to propose a five-step approach to planning data-driven digital twins of these systems and processes. A visualization is introduced for different participants. The study offers companies a guideline for defining and preparing the right parameters for different manufacturing systems and processes. After illustrating the approach useful for any simulation software tool, a case study of internal logistics (i.e., material flow) of a laboratory-scale discrete manufacturing system is presented. The case study of planning the digital twin for material logistics validated the proposed approach.

Further interesting research is presented in "A Study on Distance Measurement Module for Driving Vehicle Velocity Estimation in Multi-Lanes Using Drones" by KwanHyeong Lee, wherein a distance measurement module was developed to acquire driving vehicle information with safe and efficient mobility in multi-lanes, simultaneously with a single system, using a drone system. The authors illustrate the developed module, composed of six LiDAR sensors, which measures the distance between the driving vehicle and the drone and the vehicle passing time between two measurement points on the road. Through an experiment which compares the velocity accuracy of the drone driving vehicle with the speed gun measurement, the best efficiency of the developed drone with respect to the existing driving vehicle measurement equipment is shown to acquire information on the driving vehicle and a person's safety in a dark environment.

"Product Quality Prediction for Wire Electrical Discharge Machining with Markov Transition Fields and Convolutional Long Short-Term Memory Neural Networks" by JehnRuey Jiang and Cheng-Tai Yen describes a method for WEDM product quality prediction. The WEDM workpiece surface roughness was studied right after manufacturing with the MTF model and the CLSTM neural network using static machining parameters and dynamic manufacturing conditions. The authors illustrate the experiments conducted to evaluate the MTF-CLSTM performance via comparison with related research.

The manuscript "Research on Fish Slicing Method Based on Simulated Annealing Algorithm" by Shuo Liu, Hao Wang, and Yong Cai deals with the study of multi-objective optimization problems of irregular objects in the field of aquatic product processing. A simulated annealing algorithm was developed to solve the problem according to the characteristics of the object itself. By optimizing the mutation strategy, the ability of the simulated annealing algorithm to jump out of the local optimal solution is improved. The experimental results show that, compared with the traditional sequential algorithm method, the simulated degradation algorithm proposed in this paper effectively improves the quality of the target solution and greatly enhances the economic value of the product by addressing the multi-objective optimization problem of squid cutting.

In the manuscript "Secure and Privacy-Respecting Documentation for Interactive Manufacturing and Quality Assurance" by Paul Georg Wagner, Christian Lengenfelder, Gerrit Holzbach, Maximilian Becker, Pascal Birnstill, Michael Voit, Ali Bejhad, Tim Samorei and Jürgen Beyerer the focus is to describe use cases for automated documentation and an exemplary system architecture of a workflow recognition and documentation system. Also it derivers privacy protection goals that authors address with a suitable security architecture based on hybrid encryption, secret-sharing among multiple parties and remote attestation of the system to prevent manipulation. They finally contribute an outlook towards problems and possible solutions with regards to information that can leak through accessible metadata and with regard to more modular system architectures, where more sophisticated remote attestation approaches are needed to ensure the integrity of distributed components.

The manuscript "Visioning the Future of Smart Fashion Factories Based on Media Big Data Analysis" by Sae-Eun Lee, Naan Ju, and Kyu-Hye Lee suggests the future direction of the low-maturity smart factory in the fashion industry through newspaper analysis. In this study, semantic network analysis and convergence of iterated correlation (CONCOR) analysis were performed on 15523 news articles. The analyses revealed that the smart fashion factory was developing to incorporate automated, unmanned, and intelligent 
operation. The problem of job loss owing to the smart factory was also heavily addressed in the news articles. In the newspaper articles, the view that the smart factory is efficient, fast, and innovative, and concerns regarding the possible damages that will result from hacking and machine malfunction were simultaneously expressed. Therefore, if news about security improvement emerges in the future, negative public opinion will be reduced, positively influencing the government's support for smart factories and policy making.

Also in the manuscript "Research on Temperature Compensation Method in Crankshaft Online Measurement System" by Tingting Gu, Xiaoming Qian and Peihuang Lou a crankshaft online measurement system has realized the full inspection function with fast beats, at the same time it requires for high-precision measurement. Considering the effect of ambient temperature and temperature changes on measuring machine, the calibration part, the measured crankshaft and displacement sensor, a temperature compensation method is proposed. Firstly, relationship between calibration part and ambient temperature can be get through the zero calibration. Then use the material properties to obtain compensation values of the calibration part and the measured crankshaft part at different temperatures. Finally, the compensation parameters for displacement sensor can be obtained through the BP algorithm.

Finally, the manuscript "HSV Color-Space-Based Automated Object Localization for Robot Grasping without Prior Knowledge" by Hyun-Chul Kang, Hyo-Nyoung Han, HeeChul Bae, Min-Gi Kim, Ji-Yeon Son and Young-Kuk Kim proposes a simple and robust HSV color-space-based algorithm that can automatically extract object position information without human intervention or prior knowledge. The proposed algorithm, which does not require users to reset the HSV color threshold value whenever a product is changed, uses ROI referencing method to solve this problem. The algorithm automatically identifies the object's location by using the HSV color-space-based ROI random sampling, ROI similarity comparison, and ROI merging. The proposed system utilizes an IoT device with several modules for the detection, analysis, control, and management of object data.

Funding: This research received no external funding.

Institutional Review Board Statement: Not applicable.

Informed Consent Statement: Informed consent was obtained from all subjects involved in the studies.

Acknowledgments: This research was supported by the Università degli Studi di Catania within the CRUI-CARE Agreement (Research Path PIA.CE.RI. 2020-2022 Linea 4) and Starting Grant 2020-2022 Linea 3-Progetto NASCAR-Prot. 308811.

Conflicts of Interest: The author declares no conflict of interest.

\section{References}

1. Petrillo, A.; De Felice, F.; Cioffi, R.; Zomparelli, F. Fourth industrial revolution: Current practices, challenges, and opportunities. In Digital Transformation in Smart Manufacturing; IntechOpen: London, UK, 2018; pp. 1-20.

2. Guo, N.; Leu, M.C. Additive manufacturing: Technology, applications and research needs. Front. Mech. Eng. 2013, 8, $215-243$. [CrossRef]

3. Zheng, P.; Wang, H.; Sang, Z.; Zhong, R.Y.; Liu, Y.; Liu, C.; Xu, X. Smart manufacturing systems for Industry 4.0: Conceptual framework, scenarios, and future perspectives. Front. Mech. Eng. 2018, 13, 137-150. [CrossRef]

4. Resman, M.; Pipan, M.; Šimic, M.; Herakovič, N. A new architecture model for smart manufacturing: A performance analysis and comparison with the RAMI 4.0 reference model. Adv. Prod. Eng. Manag. 2019, 14, 153-165. [CrossRef]

5. Calì, M.; Pascoletti, G.; Gaeta, M.; Milazzo, G.; Ambu, R. A new generation of bio-composite thermoplastic filaments for a more sustainable design of parts manufactured by FDM. Appl. Sci. 2020, 10, 5852. [CrossRef]

6. Pascoletti, G.; Calì, M.; Bignardi, C.; Conti, P.; Zanetti, E.M. Mandible morphing through principal components analysis. In International Conference on Design, Simulation, Manufacturing: The Innovation Exchange; Springer: Cham, Switzerland, 2019; pp. 15-23.

7. Ambu, R.; Motta, A.; Calì, M. Design of a customized neck orthosis for FDM manufacturing with a new sustainable bio-composite. In International Conference on Design, Simulation, Manufacturing: The Innovation Exchange; Springer: Cham, Switzerland, 2019; pp. 707-718. 